\title{
Prediction of missile spare parts consumption based on time series
}

\author{
Xiaowei Ma a , Liuying Wang and Tao WANG \\ Xi'an High Technology Institute, Xian 710025, China; \\ a791807068@qq.com
}

Keywords: Missile spares, Consumption forecast, Time series.

\begin{abstract}
The scientific management of missile spare parts is to improve the efficiency of equipment management, improve equipment readiness, one of the core contents of recovery and improve the battle effectiveness of the army. At present, missile spare parts management and prediction lacked the work which based on the analysis and prediction model of missile spare parts consumption time. So, time characteristics of missile spare parts are analyzed, and Correlation prediction models are put, which improving the level of missile spare parts protection.
\end{abstract}

\section{Introduction}

Now, Type of missile equipment spare parts, quantity, involving multiple types and distribution is very scattered, due to the lack of effective means of advanced methods, the application of spare parts procurement, maintenance, replacement, stock and supply mainly rely on manpower and experience, there is no accurate and comprehensive information support, lack of scientific statistics and analysis. The sharing of information is difficult, the management efficiency is low and serious waste .

In the research of domestic spare parts, the navy is more in-depth in the field, the main research institutions are Naval Engineering University and Naval Aeronautical Engineering Institute. Naval University of Engineering Li Dawei made the initial scheme of spare parts adjustment based on Bayes method $^{[1]}$, Ruan Minzhi established a spare parts suitable for arbitrary structure system optimization model to meet the rate of spare parts allocation ${ }^{[2]}$; Wang Rui put forward the combat unit for dynamic task two grade two level repairable spare parts optimization method ${ }^{[3]}$; Li Qingmin proposed the optimization method of structure under arbitrary configuration of repairable spare parts ${ }^{[4]}$; Liu Tianhua proposed an improved algorithm for Weibull type of spare parts demand ${ }^{[5]}$; Zhao Jianzhong of Naval Aeronautical Engineering Institute was proposed based on rough set and entropy and the improved missile spares support vector machine consumption prediction method ${ }^{[6]}$.

In addition, Wang Zhengyuan put forward the spare support rate based model of carrying spares ${ }^{[7]}$; Tao Xiaochuang established a prediction model of the demand for spare parts spare support probability distribution ${ }^{[8]}$; Liu Xichun put forward the demand of wartime spare parts prediction method based on fuzzy reasoning ${ }^{[9]}$; Hong Dongpao proposed method for LRU missile based on spare parts planning mission profile ${ }^{[10]}$; Xu Xiaoyan proposed a classification based on the demand characteristics of the spare parts inventory management method and empirical research ${ }^{[11]}$; Nie Tao proposed equipment spare parts two echelon closed loop supply chain inventory optimization and analysis ${ }^{[12]}$.

Study abroad, Busacca proposed building supply security system model based on random number Monte Carlo, using the heuristic optimization algorithm to find the optimal inventory; Oliveto model is put forward based on optimizing spare parts availability, to improve equipment availability; Engaged in related research of Carnegie Mellon University professor Heiling computer team leadership in the engineering construction and data for massive data analysis; Stevens Institute of Technology professor Sauser to undertake a number of research topics related to the U.S. Department of defense, the U.S. Army mainly carried out in the research of supply chain management, inventory optimization.

The part of study at home and abroad to carry out the above focus on application, focuses on the theory, many assumptions required, according to the available data is less, the related research work, and the lack of analysis and prediction model of Missile Based on the consumption of spare parts based 
on the characteristics of the time, according to the time characteristic of missile spare parts are analyzed and put forward the relevant forecast model, improve the security level of missile spare parts.

\section{Characteristic analysis of spare parts data}

Taking a certain type of missile weapon equipment used as 'hydraulic oil' as an example, the first quarter of 2011 to the first quarter of 2016, spare parts data analysis. Figure 1:

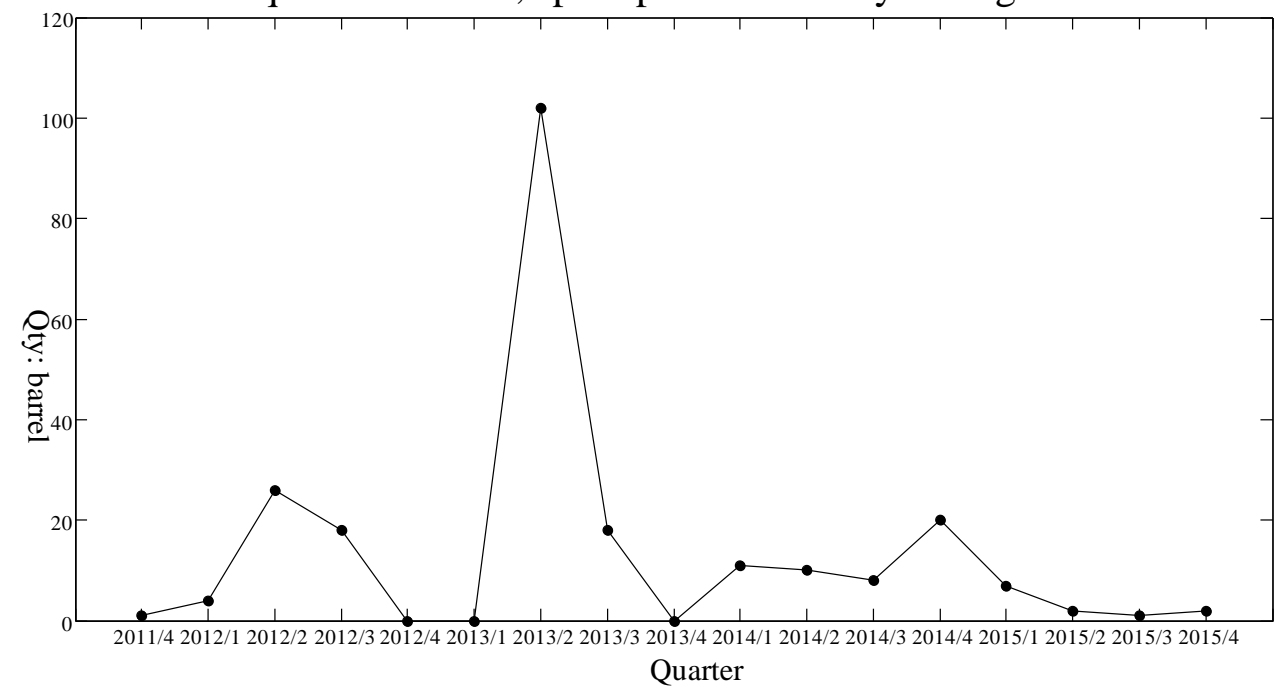

Fig. 1 Hydraulic oil consumption

Analysis of Figure 1, missile equipment spare parts usage information, we found that two spare parts historical data showing the laws of a certain time, can be regarded as a set of time series, so we can sequence correlation theory to build the prediction model of spare parts demand by the time of the relevant data for processing and analysis.

\section{Time series forecasting model for spare parts data prediction}

The characteristic parameters of the predictor variables collected by $\mathrm{N}$ are recorded as a non-stationary random process which using non stationary time series to forecast the demand of spare parts. The mathematical model is

$\mathbf{X}_{t}=\mathbf{Y}_{t}+\mathbf{Z}_{t}$

$\mathbf{Y}_{t}$ is a trend time series component of $\mathbf{X}_{t} \cdot \mathbf{Y}_{t}$ is the basis for the establishment of the degradation trajectory model which reflects the general trend of the forecast variables of missile equipment spare parts consumption rule. $\mathbf{Z}_{t}$ is a stochastic time series component of $\mathbf{X}_{t}$ which reflects the randomness of forecasting variables. Therefore, the mathematical model of the missile equipment spare parts consumption law includes the following two parts, one is mathematical model for time series prediction of trend, another is Mathematical model for time series prediction of random items.

\subsection{Establishment of time series forecasting model of trend term.}

The test data of the characteristic parameters of the prediction model of missile spare parts consumption rule is $\mathrm{X}_{\mathrm{N}}$. According to the characteristics and trend of test data, regression analysis is used to directly fit the data. The number of action $\mathrm{N}$ is a regression variable. $\mathrm{X}$ is a dependent variable. A mathematical model for time series prediction of trend term is built by regression variable and dependent variable. $X_{N}$, the test data of some characteristic parameters of prediction model for missile equipment spare parts consumption law is represented by the relative time.

$$
t_{0}=0, h=1, t_{k}=t_{0}+h k=k \quad(k=1,2, \cdots N)
$$

The time series prediction model of trend term is generally described as

$$
\begin{aligned}
Y_{t}=Y\left(t_{k}\right)= & C_{0}+C_{1} t_{k}+C_{2} t_{k}^{2}+C_{3} t_{k}^{3}+C_{4} t_{k}^{4}+C_{5} t_{k}^{-1}+C_{6} t_{k}^{-2}+ \\
& C_{7} t_{k}^{\frac{1}{2}}+C_{8} t_{k}^{-\frac{1}{2}}+C_{9} a^{t_{k}}+C_{10} t_{k}^{b}+C_{11} \ln t_{k}
\end{aligned}
$$


$a, b, C_{j} \quad(j=1,2, \cdots 11)$ are all undetermined parameters.

The test data $X_{1}, X_{2}, \cdots, X_{N}$, using the least squares method to estimate the regression parameters, can get $\hat{C}_{j} \quad(j=1,2, \cdots 11)$ which is the estimated value of $C_{j} \quad(j=1,2, \cdots 11)$, then the estimated value of $Y_{t}$ can be obtained.

$$
\begin{aligned}
\hat{Y}_{t}=\hat{Y}\left(t_{k}\right)= & \hat{C}_{0}+\hat{C}_{1} t_{k}+\hat{C}_{2} t_{k}^{2}+\hat{C}_{3} t_{k}^{3}+\hat{C}_{4} t_{k}^{4}+\hat{C}_{5} t_{k}^{-1}+\hat{C}_{6} t_{k}^{-2}+ \\
& \hat{C}_{7} t_{k}^{\frac{1}{2}}+\hat{C}_{8} t_{k}^{-\frac{1}{2}}+\hat{C}_{9} a^{t_{k}}+\hat{C}_{10} t_{k}^{b}+\hat{C}_{7} \ln t_{k}
\end{aligned}
$$

In order to measure the pros and cons of the above regression equation to fit the observed data, the residual square sum SSE was chosen as the optimal regression model. The expression is

$$
S S E=\sum_{k=1}^{N}\left(\hat{Y}_{t}\left(t_{k}\right)-Y_{t}\left(t_{k}\right)\right)^{2}
$$

The smaller the value, the smaller the difference between the fitting value and the observed value, and the more accurate the function fit.

\subsection{Establishment of Multivariate Forecasting Model for Time Series of Random Items.}

The stochastic term is regarded as a time series. An important prerequisite is to ensure that the sequence is a zero mean stationary time series. Therefore, the zero mean value test and the stability test.

(1) Zero mean test

For zero mean test, is to test $H_{0}: \mu=0$. Statistic $T=\bar{x} \sqrt{n} / s . \bar{x}$ is sequence mean, $s$ is standard deviation of a sequence. $n$ is sequence length. Under the assumption of $H_{0}, T$ obeys $t(n-1)$ distribution. Give significance level $\alpha(\alpha \leq 0.05)$, when $|T|<t_{\alpha / 2}(n-1)$, sequence is zero sequence.

(2) Stationarity test

This paper use the autocorrelation function $(A C F)$ and partial autocorrelation function $(P A C F)$ test.

Autocorrelation coefficient $\gamma_{k}$ can be used to measure the degree of autocorrelation, which indicates that the correlation between observations in a time series is separated from the $k$ period.

$$
\gamma_{k}=\frac{E\left(x_{t}, x_{t++}\right)}{E\left(x_{t}^{2}\right)}=\frac{\sum_{t=1}^{n-k}\left(x_{t}-\bar{x}\right)\left(x_{t+k}-\bar{x}\right)}{\sum_{t=1}^{n}\left(x_{t}-\bar{x}\right)^{2}}
$$

$n$ is sample size. $k$ is lag period. $\bar{x}$ is Sample mean. $\gamma_{k}$ range of $[-1,1]$. The closer the absolute value of $\mathrm{A}$ to 1 , the higher the degree of autocorrelation is.

Partial autocorrelation is defined as time series $\left\{x_{t}\right\}$, under certain conditions, the conditional correlation between time series. We use partial autocorrelation coefficient $\varphi_{k k}$ to measure the degree of correlation, $\varphi_{k k}$ value is $[-1,1]$.

$$
\varphi_{k k}=\left\{\begin{array}{c}
\gamma_{1} \\
\frac{\gamma_{k}-\sum_{j=1}^{k-1} \varphi_{k-1, j} \gamma_{k-j}}{1-\sum_{j=1}^{k-1} \varphi_{k-1, j} \gamma_{j}}
\end{array}\right.
$$

For a time series, the stability of the original sequence is judged according to the correlation graph. It can be determined that the time series is non-stationary. If the autocorrelation coefficient is oscillating, the time series is stationary. In practical applications, we consider the autocorrelation and partial autocorrelation functions together.

(3) Stationary time series forecasting model

For missile equipment spare parts consumption forecast, assuming that $n$ affected by the characteristic parameters of missile equipment spare parts consumption which is stationary time series 
for $\mathbf{Z}_{t}=\left(z_{1, t}, z_{2, t}, \cdots, z_{n, t}\right)^{T}, t=1,2, \cdots N$, for each component of $z_{j, t}, j=1,2, \cdots, n$, the mathematical model for forecasting :

$$
\begin{aligned}
z_{j, t}=\alpha_{j 1}^{(1)} z_{1, t-1}+\alpha_{j 1}^{(2)} z_{1, t-2}+\cdots+\alpha_{j 1}^{(p)} z_{1, t-p}+ \\
\alpha_{j 2}^{(1)} z_{2, t-1}+\alpha_{j 2}^{(2)} z_{2, t-2}+\cdots+\alpha_{j 2}^{(p)} z_{2, t-p}+ \\
\cdots+\alpha_{j n}^{(1)} z_{n, t-1}+\alpha_{j n}^{(2)} z_{n, t-2}+\cdots+\alpha_{j n}^{(p)} z_{n, t-p}+\varepsilon_{t}^{(j)}
\end{aligned}
$$

\section{Vector form}

$$
\begin{aligned}
& \mathbf{Z}_{t}=\alpha_{1} \mathbf{Z}_{t-1}+\alpha_{2} \mathbf{Z}_{t-2}+\cdots+\alpha_{p} \mathbf{Z}_{t-p}+\boldsymbol{\varepsilon}_{t} \\
& \mathbf{Z}_{t}=\left(z_{1, t}, z_{2, t}, \cdots, z_{n, t}\right)^{T} ; \quad \boldsymbol{\varepsilon}_{t}=\left(\varepsilon_{t}{ }^{(1)}, \varepsilon_{t}{ }^{(2)}, \cdots, \varepsilon_{t}{ }^{(n)}\right)^{T} \\
& \boldsymbol{\alpha}_{i}=\left[\begin{array}{cccc}
\alpha_{11}^{(\mathrm{i})} & \alpha_{12}^{(\mathrm{i})} & \cdots & \alpha_{1 n}^{(\mathrm{i})} \\
\alpha_{21}^{(\mathrm{i})} & \alpha_{22}^{(\mathrm{i})} & \cdots & \alpha_{2 n}^{(\mathrm{i})} \\
\vdots & \vdots & \ddots & \vdots \\
\alpha_{n 1}^{(\mathrm{i})} & \alpha_{n 2}^{(\mathrm{i})} & \cdots & \alpha_{n n}^{(\mathrm{i})}
\end{array}\right], i=1,2, \cdots, p
\end{aligned}
$$

Coefficient matrix $\alpha_{1}, \alpha_{2}, \cdots, \alpha_{p}$, meet the stable condition:

$$
\operatorname{det}\left(\mathbf{I}-\alpha_{1} u-\alpha_{2} u^{2}-\cdots-\alpha_{p} u^{p}\right) \neq 0 \quad(|u| \leq 1)
$$

$u$ is plural.

(4) Model parameter estimation

If the data vector of the stationary time series of the $n$ characteristic parameters of the missile equipment spare parts consumption model is $\mathbf{Z}_{t}=\left(z_{1, t}, z_{2, t}, \cdots, z_{n, t}\right)^{T}, t=1,2, \cdots N$, the $p$ order stationary linear autoregressive model can be expressed as

$$
\mathbf{Z}_{j}=\mathbf{A}_{j} \boldsymbol{\alpha}_{j}+\boldsymbol{\varepsilon}_{j}
$$

When $E\left(\boldsymbol{\varepsilon}_{j}\right)=0, E\left(\boldsymbol{\varepsilon}_{j} \boldsymbol{\varepsilon}_{j}^{T}\right)=\sigma^{2} \mathbf{I}, \hat{\boldsymbol{\alpha}}_{j}$ is a linear unbiased minimum variance estimation of $\boldsymbol{\alpha}_{j}$.

Based on the above model, we can get the prediction of spare parts consumption as long as the time series of the characteristic parameters of missile equipment spare parts consumption rule model are obtained.

\section{Example verification}

Based on a certain type of hydraulic oil as an example, a total of 17 sets of data, the first 12 sets of data as training data, after 5 sets of data as test data to verify the proposed availability of spare parts demand forecasting model based on time series.

First of all, the regression analysis of the trend items, the regression model of the $S_{t-2}$ index and the related parameters are shown in Table 1.

Table 15 function regression model of $S_{t-2}$ index trend

\begin{tabular}{|c|c|c|}
\hline Fitting Type & Regression Model & Regression Precision \\
\hline linear regression & $-0.3926 n+221.6$ & 7.225 \\
\hline polynomial regression & $-8.699 \times 10^{-7} n^{3}+0.001001 n^{2}-0.6475 n+234.6$ & 5.214 \\
\hline Power regression & $4.222 e^{-0.0323 n}+231.5 e^{-0.002578}$ & 5.209 \\
\hline Exponential regression & $361.7 n^{-0.1799}$ & 21.44 \\
\hline Hyperbolic regression & $1 /\left(-0.254 n^{2}+0.2844 n-0.0261\right)$ & 158.1 \\
\hline
\end{tabular}

The regression accuracy of various regression models is compared with the power regression model

$$
\hat{t}_{c n}=4.222 e^{-0.0323 n}+231.5 e^{-0.002578}
$$


The $S_{t-1}$ index is compared and analyzed. The results of each model are shown in table 2.

Table 25 function regression model of $S_{t-1}$ index trend

\begin{tabular}{|c|c|c|}
\hline Fitting Type & Regression Model & Regression Precision \\
\hline linear regression & $5.33 n+7880$ & 143.6 \\
\hline polynomial regression & $5.027 \times 10^{-5} n^{3}-0.03549 n^{2} 12.22 n+7585$ & 110.5 \\
\hline Power regression & $8135 e^{-0.000493 \pi}-797.7 e^{-0.0338 n}$ & 99.47 \\
\hline Exponential regression & $6373 n^{0.06621}$ & 178.5 \\
\hline Hyperbolic regression & $1 /\left(0.3905 n^{2}+0.01959 n+0.5514\right)$ & 8871 \\
\hline
\end{tabular}

$S_{t-1}$ trend term time series model:

$$
\hat{t}_{x n}=8135 e^{-0.000493 \pi}-797.7 e^{-0.0338 n}
$$

Based on the demand of spare parts, the vector matrix of the stationary time series with $S_{t-2}$ and $S_{t-1}$ as independent variables is further fitted.

$$
\mathbf{Z}_{t}=\alpha_{1} \mathbf{Z}_{t-1}+\alpha_{2} \mathbf{Z}_{t-2}+\alpha_{3} \mathbf{Z}_{t-3}+\alpha_{4} \mathbf{Z}_{t-4}
$$

$\mathbf{Z}_{t}=\left(z_{1, t}, z_{2, t}\right)$ is the prediction vector. Its parameters are estimated as:

$$
\begin{aligned}
\alpha_{1} & =\left[\begin{array}{cc}
-0.0416417156668 & 1.88497963101 \times 10^{-5} \\
-1.03626894403 & 0.48700901924
\end{array}\right] \\
\alpha_{2} & =\left[\begin{array}{ll}
0.0331975129198 & 5.35661501552 \times 10^{-5} \\
0.584799489882 & 0.399405321603
\end{array}\right] \\
\alpha_{3} & =\left[\begin{array}{cc}
0.0737525382487 & -0.00456845765106 \\
0.224042592928 & -0.00471814140297
\end{array}\right] \\
\alpha_{4} & =\left[\begin{array}{ll}
-0.105006777786 & 0.00468892597472 \\
-0.187794249458 & -0.00433463070703
\end{array}\right]
\end{aligned}
$$

The mathematical model for predicting the double variable consumption of spare parts demand is

$$
\begin{aligned}
{\left[\begin{array}{l}
z_{1, t} \\
z_{2, t}
\end{array}\right]=} & {\left[\begin{array}{cc}
4.222 e^{-0.0323 n}+231.5 e^{-0.002578 n} \\
8135 e^{-0.0004937 n}-797.7 e^{-0.03381 n}
\end{array}\right]+} \\
& {\left[\begin{array}{cc}
-0.04164 & 1.8850 \times 10^{-5} \\
-1.0363 & 0.4870
\end{array}\right]\left[\begin{array}{l}
z_{1, t-1} \\
z_{2, t-1}
\end{array}\right]+} \\
& {\left[\begin{array}{cc}
0.03319 & 5.3566 \times 10^{-5} \\
0.5847 & 0.3994
\end{array}\right]\left[\begin{array}{l}
z_{1, t-2} \\
z_{2, t-2}
\end{array}\right]+} \\
& {\left[\begin{array}{cc}
0.07375 & -0.004568 \\
0.2240 & -0.004718
\end{array}\right]\left[\begin{array}{l}
z_{1, t-3} \\
z_{2, t-3}
\end{array}\right]+} \\
& {\left[\begin{array}{cc}
-0.1051 & 0.004689 \\
-0.1878 & -0.004335
\end{array}\right]\left[\begin{array}{l}
z_{1, t-4} \\
z_{2, t-4}
\end{array}\right] }
\end{aligned}
$$

To verify the remaining 5 groups of data, the verification results are shown in Figure 2. 


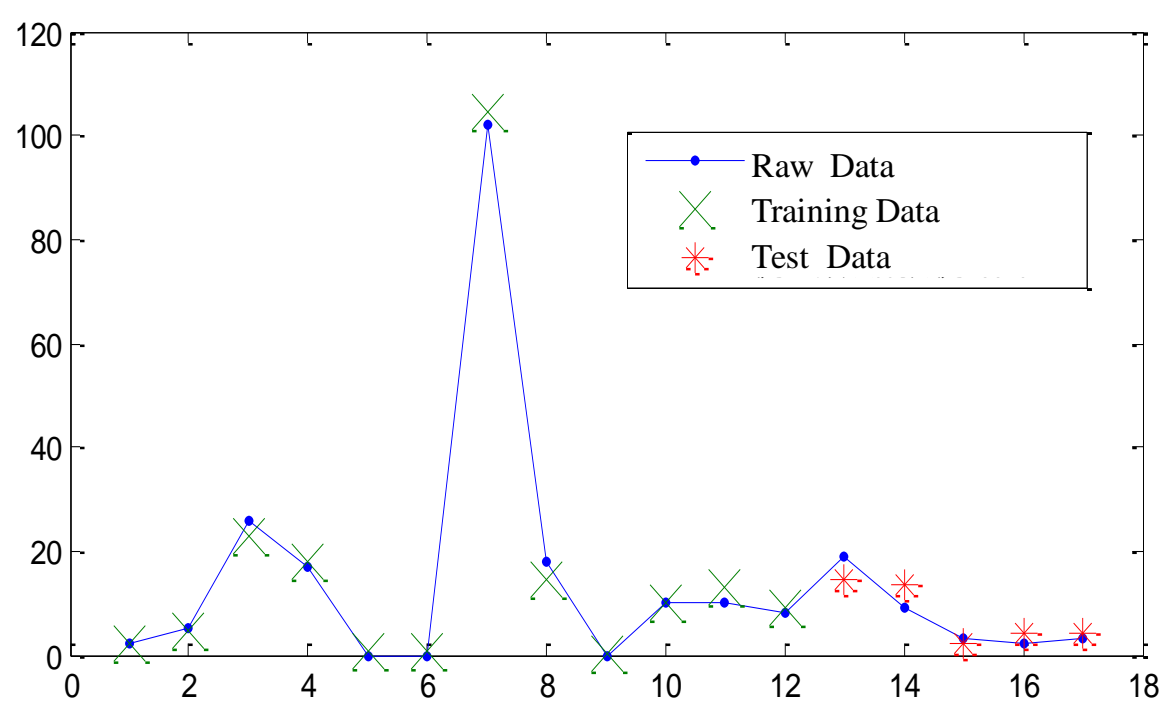

Fig. 2 Part of the data analysis of a certain type of hydraulic oil

Table 3 gives the prediction results of the data of the five kinds of spare parts, which shows that the prediction accuracy is more accurate, and can meet the demand of the forecast of the demand of the five kinds of spare parts.

Table 3 Prediction results of the data of 5 kinds of spare parts

\begin{tabular}{|c|c|c|c|c|c|c|c|c|}
\hline Spare name & \multirow{2}{*}{$\begin{array}{c}\text { Training } \\
\text { data }\end{array}$} & \multirow{2}{*}{$\begin{array}{c}\text { Test } \\
\text { data }\end{array}$} & \multicolumn{2}{|c|}{ Training data } & \multicolumn{2}{|c|}{ Test data } & \multicolumn{2}{c|}{ All data } \\
\cline { 5 - 9 } & & & error & accuracy & error & accuracy & error & accuracy \\
\hline Hydraulic oil & 12 & 5 & 3.44 & $82.48 \%$ & 9.85 & $68.24 \%$ & 5.33 & $78.29 \%$ \\
\hline Diesel filter & 7 & 3 & 5.31 & $78.89 \%$ & 13.50 & $73.16 \%$ & 10.60 & $77.17 \%$ \\
\hline Oil filter & 9 & 4 & 9.72 & $72.20 \%$ & 19.20 & $60.28 \%$ & 12.64 & $68.22 \%$ \\
\hline Air filter & 6 & 3 & 11.11 & $68.92 \%$ & 15.69 & $67.82 \%$ & 12.64 & $68.55 \%$ \\
\hline $\begin{array}{c}\text { Four circuit protection } \\
\text { valve }\end{array}$ & 8 & 4 & 0.18 & $88.23 \%$ & 1.38 & $88.86 \%$ & 0.54 & $88.44 \%$ \\
\hline
\end{tabular}

\section{Summary}

The forecasting model of spare parts consumption based on time series has generality and universality. Because the data of missile spare parts have the characteristics of time series, the time series model is widely applicable to various types of weapon equipment spare parts. The prediction results of typical spare parts basically meet the requirements. Although with less data and typical parts with missing data, but the time series model for forecasting accuracy of the training data and the testing data were above $60 \%$, can basically meet the demand forecast for typical parts. Spare parts consumption has obvious task characteristics. That is, peacetime training has a relatively stable demand for spare parts, when the implementation of major tasks, there is a significant temporary demand, so the next step according to the needs of peacetime and wartime needs to distinguish.

\section{References}

[1].LI Da-wei, ZHANG Zhi-hua. The evaluation of operational reliability based on information of spare part demand. Systems Engineering-Theory \& Practice. Vol. 31 (2011) No. 9, p. 1770-1775. 
[2]. RUAN Wen-zhi, LI Qing-min, PENG Ying-wu, et al. Model of spare part fill rate for systems of various structures and optimization method. Systems Engineering and Electronics. Vol. 33 (2011) No. 8, p. 1799-1803.

[3]. WAGN Rui, PENG Ying-wu, LI Qing-min, et al. Dynamic mission-oriented two-echelon and two-indenture repairable spare parts optimization for combat units. Systems Engineering and Electronics. Vol. 35 (2013) No. 4, p. 761-769.

[4]. ZHOU Liang, LI Qing-min, WAGN Rui, et al. Spare parts allocation with ship for K/N(G) redundant structure. Systems Engineering and Electronics. Vol. 37 (2015) No. 12, p. 2785-2790.

[5]. LIU Tian-hua, ZHANG Zhi-hua, LIANG Sheng-jie, et al. An improved method for the spare demand of the Weibull-distribution. Systems Engineering-Theory \& Practice. Vol. 32 (2012) No. 5, p. 1124-1128.

[6]. ZHAO Jian-zhong, XU Ting-xue, LIU Yong, et al. Consumption Forecasting of Missile Spare Parts Based on Rough Set, Entropy Weight and Improved SVM. ACTA ARMAMENTARII. Vol. 33 (2012) No. 10, p. 1258-1265.

[7]. WANG Zheng-yuan, LI Jin, ZHU Yu, et al. Spare Supporting Rate Based Model of Carrying Spares. ACTA ARMAMENTARII. Vol.27 (2006) No. 6, p. 1136-1139.

[8]. TAO Xiao-chuang, GUO Lin-han, XIAO Bo-ping, et al. Demand Prediction Model for Spare Parts Based on Fill Rate Allocation. ACTA ARMAMENTARII. Vol. 33 (2012) No. 8, p. 975-979.

[9]. LIU Xi-chun, ZHU Long-shi, ZHANG We. Demand Forecasting of Fuzzy Inference-based Wartime Spares. ACTA ARMAMENTARII. Vol. 34 (2013) No. 9, p. 1197-1200.

[10]. HONG Dong-pao, XU Nuo, JIN Jing, et al. LRU spare parts programming for missile based on mission profile. Systems Engineering and Electronics. Vol. 37 (2015) No. 11, p. 2520-2523.

[11]. XU Xiao-yan. A Demand Classification Based Approach of Inventory Management for Spare-parts and Its Application. Systems Engineering-Theory \& Practice. No. 2 (2006), p. 62-67.

[12]. NIE Tao, SHENG Wen, WANG Han-zhong. Optimizing and analyzing two-echelon closed loop supply chain storage system for equipment spare parts. Systems Engineering-Theory \& Practice. Vol. 30 (2010) No. 12, p. 2309-2314. 